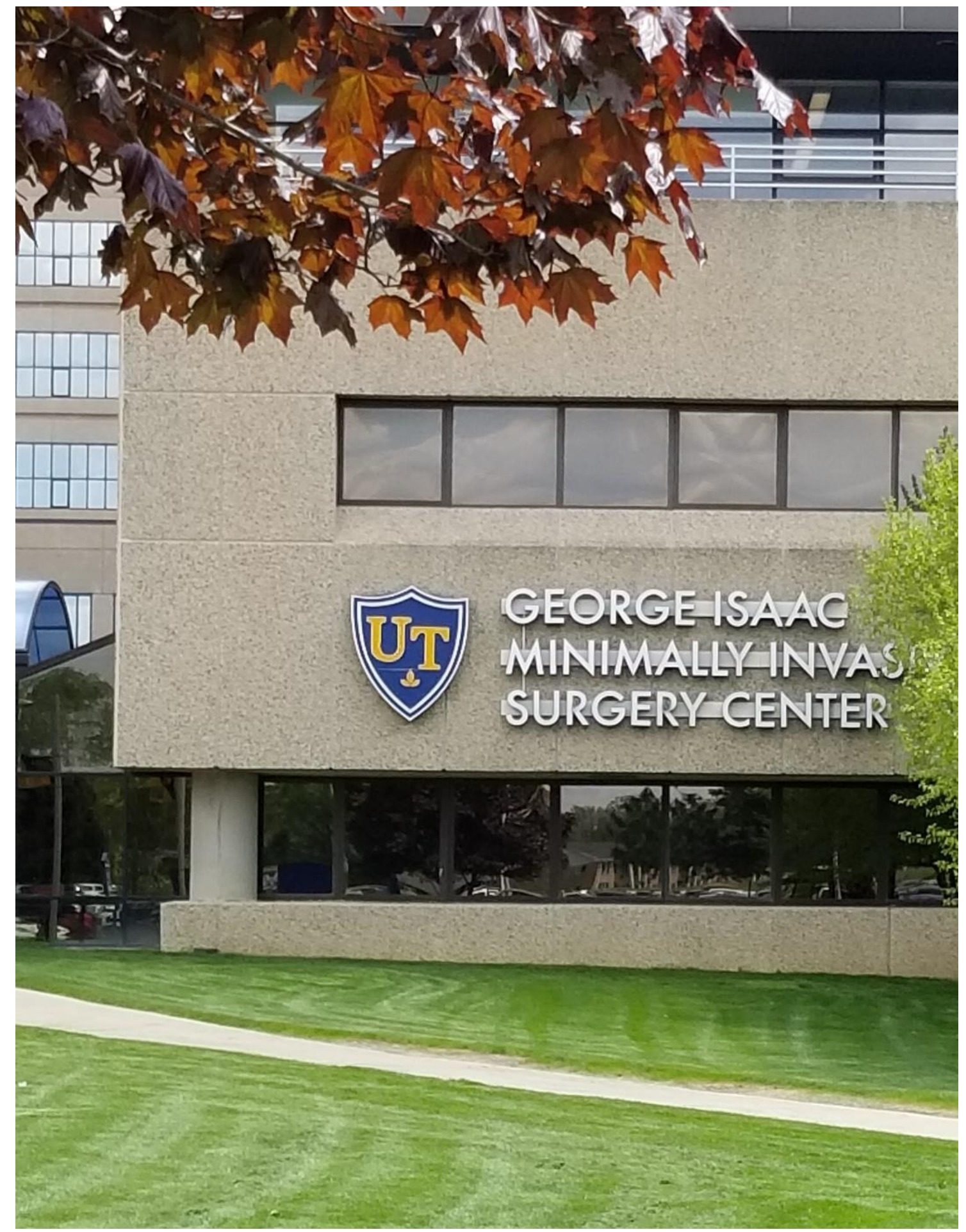




\title{
Acute bilateral ischemic stroke in a young adult without risk factors
}

\author{
Maisa Alafyouni ${ }^{a}, 1$ Derrick Huang ${ }^{b, 1}$ Scott Kleiman ${ }^{c}$ and Shanna Jones ${ }^{b}$
}

${ }^{a}$ Department of Emergency Medicine, Royal Oak, MI, USA, ${ }^{b}$ Oakland University, William Beaumont School of Medicine, Rochester, MI, USA, and ${ }^{c}$ Department of Emergency Medicine, Troy Beaumont Hospital, Troy, MI, USA

\begin{abstract}
Emergency department (ED) management of cerebrovascular accidents (CVA) in younger patients is complicated by atypical presentations, rarer etiologies, and greater disability costs. A previously healthy 27-year-old woman presented to the ED for a trauma resuscitation that was subsequently converted into a stroke resuscitation with a last known normal of 8 hours. Computed tomography perfusion scan results diagnosed the patient with a bilateral ischemic stroke that was successfully treated with a mechanical thrombectomy. The case highlights the need for a high index of suspicion in younger CVA patients whose greater potential for atypical presentations may compromise time-sensitive treatments.
\end{abstract}

| young stroke | bilateral CVA | thrombectomy | post-partum | perfusion scan |

C erebrovascular accidents (CVA) are currently the fifth leading cause of death in the United States (1). In the emergency department (ED), acute ischemic stroke was the primary diagnosis in over 600,000 ED visits each year from 2010 to 2013, with over $90 \%$ of these visits resulting in admission or transfer to another hospital (2).

CVAs are also a major burden in the US from both a quality of life and disability standpoint as well as an economic burden (2). This is particularly problematic for younger CVA patients, often defined as those aged 18 to 54, who may be disabled during their most productive years of life (3). Although CVAs in this patient group are relatively rare, making up only $16 \%$ of all CVAs in 2013, incidence of this disease among ED visits continues to remain stable (2). This is reflected in national trend data revealing that the ED visit rate for ischemic stroke or TIA for patients aged 55 and older has decreased, whereas no change was observed for patients aged 18 to 54 , highlighting a persistent concern for care providers $(1,2$, 4).

Time-sensitive decision making and the undifferentiated nature of patients in the ED are inherent in the assessment and treatment of CVA patients. In younger patients, this difficulty is exacerbated by atypical presentations, such as headache and dizziness, as well as potentially rarer etiologies, such as antiphospholipid antibody syndrome and hyperhomocysteinemia (5-7). Furthermore, CVAs may be misdiagnosed as seizures and the sequelae of CVAs may themselves precipitate traumatic accidents, further obscuring assessment. These difficulties complicate differential diagnoses and result in the potential for misdiagnosis and delayed CVA treatment $(5,8)$. Here, we present a case that showcases the persistent complexities of CVA care in a young patient and involves new imaging modalities in the $\mathrm{ED}$ that are essential in diagnosis and subsequent treatment.

\section{Case report}

Patient information. Age: 27 years, gender: female, ethnicity: caucasian. Related medical problems: 4 months post-partum, obesity, ischemic CVA.

Objective. Discuss complexities of CVA care in young patients and new imaging modalities essential in diagnosis and subsequent treatment.

Case. A previously healthy 27-year-old woman four months post-partum presented to the ED due to a motor vehicle collision (MVC). Per emergency medical services (EMS), the patient was a restrained driver traveling at 35-40 $\mathrm{mph}$ when her vehicle experienced a broadside collision with another car, resulting in damage to the front right passenger side with air bag deployment. Witnesses note that the patient had crossed a red light without applying the brakes. The patient was found covered in vomit, diaphoretic, and only responsive to pain. EMS reported that she may have had a seizure while driving.

Her husband reported that the patient has been healthy with no history of seizures, intravenous drug abuse, hypertension, hyperlipidemia, blood clots, nor bleeding disorders. He also denied any surgical and family history in the patient as well as any current medication use. The patient was last seen in her usual state of health 8 hours prior to the MVC. In the ED, the patient vomited once. Initial vitals indicated a blood pressure of $152 / 86 \mathrm{mmHg}$ with an oxygen saturation of $100 \%$ on nasal cannula, and were otherwise normal. On exam, the patient was in no apparent distress with a Glasgow Coma Scale total of $8($ Eye $=4$, Verbal $=1$, and Motor $=3)$. Her head and body were without obvious external signs of trauma and a C-collar was in place by EMS. The patient had a regular rate and rhythm, equal pulses bilaterally, no focal abdominal tenderness, and her lungs were clear to auscultation. Skin exam was normal. On neurological exam, she spontaneously withdrew her left upper and left lower extremity to pain and did not move her right upper or right lower extremity prompting a stat non-contrast computed tomography $(\mathrm{CT})$ of the head.

Initial laboratory studies showed a mild leukocytosis of 11.3 $10^{3} \mu \mathrm{l}$, glucose of $157 \mathrm{mg} / \mathrm{dl}$, and were otherwise normal - including a negative pregnancy test, urinalysis, metabolic panel, coagu-

All authors contributed to this paper. ${ }^{1}$ To whom correspondence should be sent: Maisa Alafyouni: maisa.alafyouni@beaumont.org or Derrick Huang: derrickhuang@oakland.edu

The authors declare no conflict of interest. Submitted: 08/09/2019, published: 10/10/2019.

Freely available online through the UTJMS open access option 
lation panel, and drug panel. Non-contrast CT scan of the head showed hypodense lesions in the right temporoparietal region concerning for subacute ischemia. A CT perfusion of the head was then ordered and showed occlusion of the left middle cerebral artery (MCA) M1 segment with a core infarct involving the basal ganglia mid frontal lobe, insular cortex, and the anterior temporal lobe. Ischemic penumbra was seen more distally and superiorly at the parietal lobe indicating collateralization from the anterior and posterior cerebral artery territories (see Fig. 1 and 2). There was also a right temporal lobe perfusion abnormality suggesting distal branch occlusion with ischemia. National Institutes of Health Stroke Scale was calculated at 23 .

Given the last known normal of the patient, tissue plasminogen activator (tPA) was not deemed an appropriate treatment option. Thrombectomy was considered based on the CT perfusion results and consultation with neurointerventional radiology. The patient was given a full dose aspirin and ticagrelor, intubated for airway protection, and admitted for emergent endovascular intervention. She underwent an uncomplicated mechanical thrombectomy and ultimately returned to near baseline function with a mild impairment in her language skills. All hematologic workup during her admission was negative and no clear cause was found.

\section{Discussion}

We report a case of a successful emergent endovascular intervention for bilateral MCA CVAs in a previously healthy 27 -yearold patient. This patient initially presented as a trauma resuscitation that was subsequently converted into a stroke resuscitation after clinical examination. According to the 2018 American Heart Association (AHA) and American Stroke Association (ASA) Early Management Acute Ischemic Stroke guidelines, IV tPA is provided to eligible acute stroke patients ages $>18$ within 3 hours of last known normal or within 4.5 hours of last known normal based on ECASS III exclusion criteria (9). Our patient was involved in a traumatic accident and also arrived outside of the treatment window with a last known normal of 8 hours prior to ED arrival, thus IV tPA was deemed inappropriate. Potential candidates for mechanical thrombectomy may receive a CT angiogram or MR (magnetic resonance) angiogram to identify large vessel occlusion amenable to surgical treatment. Additionally, these patients have until recently required presentation within 6 hours of last known normal. In February 2018, AHA/ASA guideline changes expanded the eligibility for mechanical thrombectomy based on the DAWN or DEFUSE 3 trials (9). In patients with clinical evidence of a large vessel occlusion in the anterior cerebral circulation presenting within 6-24 hours of last known normal, perfusion imaging (CT or MR) or MRI (magnetic resonance imaging) with DWI (diffusion weighted imaging) sequence can be ordered to evaluate for evidence of large vessel occlusion in the proximal anterior circulation. Incorporating our CT perfusion scan results in conjunction with neurointerventional radiology consultation, our patient was deemed an appropriate candidate for mechanical thrombectomy.

Classic risk factors for ischemic stroke in young patients mirror those of older adult patients, including hypertension, dyslipidemia, and cigarette smoking (10). Although both groups share identical modifiable risk factors, heart disease such as atrial fibrillation and diabetes mellitus is more prevalent in patents whereas dyslipidemia and smoking are more prevalent in younger stroke patients (10). Other more nuanced risk factors in the younger population include hyperhomocysteinemia and a history of migraine with aura, a risk that is significantly heightened among those who are also smokers and those using oral contraception $(10,11)$. Our patient did not possess typical risk factors for ischemic stroke and was not on oral contraceptive pills commonly associated with increased thrombotic risk. Although our patient was 4 months post-partum, this is outside the post-partum risk threshold of about 12 weeks for increased thrombotic risk, which is also more commonly related to cerebral venous thrombosis (12).

The most common etiologies of ischemic stroke differ in young patients compared to older patients. Various studies exploring ischemic stroke etiologies in young patients have utilized the TOAST criteria, which uses the following categories: large-vessel disease, small-vessel disease, cardioembolic stroke, other determined cause, and undetermined cause (10). In one review article utilizing the TOAST criteria, cardioembolic etiology was the most common cause of ischemic stroke in young adults, defined at under age 49, and was determined as the general etiology in $575(17.3 \%)$ of ischemic strokes in the study population of 3331 ischemic stroke patients $(3,10,13)$.

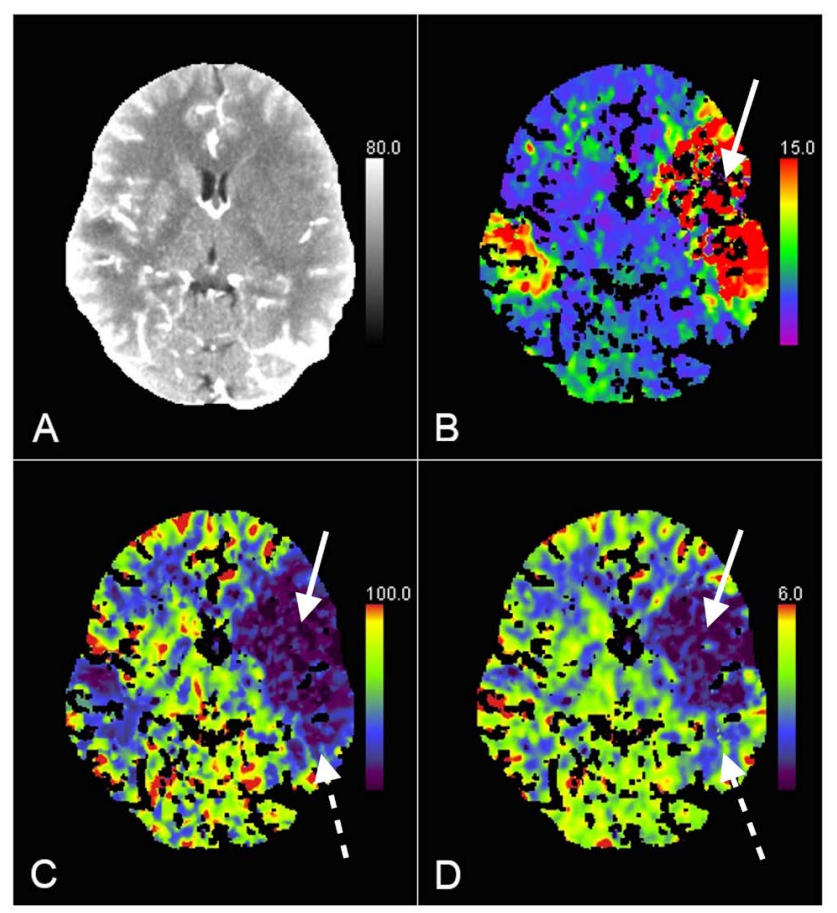

Fig. 1. Computed tomography (CT) scan with contrast (A) and CT perfusion parametric maps with time to peak $(B)$, cerebral blood flow $(C)$, and cerebral blood volume $(D)$, demonstrate occlusion of the left middle cerebral artery M1 segment with a core infarct (arrow) involving the basal ganglia mid frontal lobe, insular cortex, and the anterior temporal lobe. Ischemic penumbra (dashed arrow) was seen more distally at the parietal lobe indicating collateralization from the anterior cerebral artery and posterior cerebral artery territories. By convention, all color maps are coded red for higher values and blue for lower values.

Cardioembolic etiology included a range of pathologies including atrial fibrillation, cardiomyopathy, and valve abnormalities. In the same study population, cervicocephalic arterial dissection was the most common single etiology of ischemic stroke, comprising $426(12.8 \%)$ of cases (13). Carotid artery dissections may occur in the setting of trauma, such as strangulation and in an MVC, and the traumatic event may precede the onset of initial symptoms from several hours to days (14). Dissections may also occur spontaneously in the absence of identified trauma. Notably, outside of hyperhomo- 
cystinuria and antiphospholipid antibody syndrome, inherited coagulation disorders have not been shown to have a significant role in stroke in young patients $(7,10)$. Unfortunately, as in our case, undetermined etiology has been the most prevalent "diagnosis" in most studies using the TOAST criteria. This category includes patients with two or more potential etiologies, a negative work up, and incomplete investigation or loss to follow up (3).

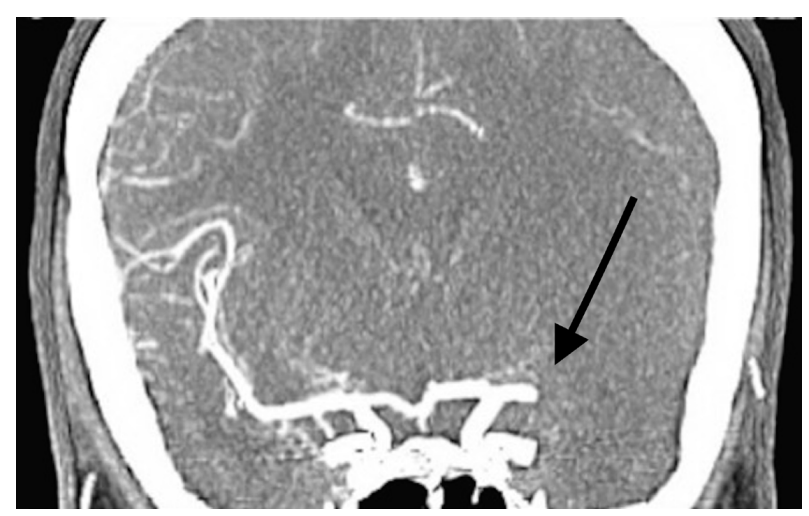

Fig. 2. Computed tomography scan with contrast coronal image demonstrating loss of cerebral blood flow to the left middle cerebral artery (black arrow).

\section{Conclusion}

Incidence of young patients presenting with ischemic stroke in the ED has been stable, despite a concomitant decrease in incidence in older age groups. As in our case, an initial trauma resuscitation may crucially convert to a stroke resuscitation based solely on a comprehensive history and physical examination, which may be difficult in younger CVA patients who more often present atypically. Clinicians are advised to have a low threshold for further exploration when the history and physical examination do not align. Expansion of candidacy for emergent surgical intervention and use of newer imaging modalities has been invaluable to management.

\section{Conflict of interest}

Authors declare no conflict of interest.

\section{Authors' contributions}

MA, DH wrote the paper, SJ, SK reviewed and revised the manuscript. All authors read and approved the final document.
1. Talwalkar A and Uddin S (2015) Trends in Emergency Department Visits for Ischemic Stroke and Transient Ischemic Attack: United States, 2001\{2011. NCHS Data Brief (194):1-8.

2. Stuntz M, et al. (2017) Nationwide trends of clinical characteristics and economic burden of emergency department visits due to acute ischemic stroke. Open Access Emerg Med 9:89-96.

3. Smajlovic $D$ (2015) Strokes in young adults: epidemiology and prevention. Vasc Health Risk Manag 11:157-164.

4. Marini C, Russo T, and Felzani G (2010) Incidence of Stroke in Young Adults: A Review. Stroke Res Treat 2011.

5. Brandler E, et al. (2015) Prehospital Stroke Identification: Factors Associated with Diagnostic Accuracy. J Stroke Cerebrovasc Dis 24(9):2161-2166.

6. $\mathrm{Xu} Y$, et al. (2019) Plasma homocysteine levels may be associated with the subtypes of ischemic stroke: a meta-analysis. Int J Clin Exp Med 12(1):117-124.

7. Brey R (2005) Antiphospholipid Antibodies in Young Adults with Stroke. J Thromb Thrombolysis 20(2):105-112.
8. Newman-Toker D, et al. (2014) Missed diagnosis of stroke in the emergency department: a cross-sectional analysis of a large population-based sample. Diagnosis 1(2):155-166.

9. Powers W, et al. (2018) 2018 Guidelines for the Early Management of Patients with Acute Ischemic Stroke: A Guideline for Healthcare Professionals from the American Heart Association/American Stroke Association. Stroke 49(6).

10. Tancredi L, et al. (2013) Stroke Care in Young Patients. Stroke Res Treat 2013.

11. Bousser $M$ and Welch $K(2005)$ Relation between migraine and stroke. Am J Ophthalmol 140(6):1173.

12. Kamel H, et al. (2014) Risk of a Thrombotic Event After the 6-Week Postpartum Period. Obstet Gynecol Surv 69(7):375-376.

13. Yesilot Barlas $\mathrm{N}$, et al. (2013) Etiology of first-ever ischaemic stroke in European young adults: the 15 cities young stroke study. Eur J Neurol 20:1431-1439.

14. Ben Hassen W, et al. (2014) Imaging of cervical artery dissection. Diagn Interv Imaging 95(12):1151-61. 